\title{
A radiation condition for the 2-D Helmholtz equation in stratified media
}

\author{
Giulio Ciraolo *
}

October 31, 2018

\begin{abstract}
We study the 2-D Helmholtz equation in perturbed stratified media, allowing the existence of guided waves. Our assumptions on the perturbing and source terms are not too restrictive.

We prove two results. Firstly, we introduce a Sommerfeld-Rellich radiation condition and prove the uniqueness of the solution for the studied equation. Then, by careful asymptotic estimates, we prove the existence of a bounded solution satisfying our radiation condition.
\end{abstract}

\section{Introduction}

A classical problem in studying the Helmholtz equation

$$
\Delta u+k^{2} n(x, z)^{2} u=f, \quad(x, z) \in \mathbb{R}^{2}
$$

is that of finding a physically meaningful criterion for uniqueness of solutions. When $k$ is real (and nonzero) and $n$ is a real-valued function, the Sommerfeld radiation condition (see [So1] and [So2]) and the Rellich Theorem [Rel] are the basis for such studies. Many papers have been written to extend the Sommerfeld and Rellich radiation conditions to situation in which the index of refraction $n$ has special properties. If the refraction index tends to a constant $n_{\infty}$ in all directions (with an appropriate behaviour), the usual uniqueness assumption is given by the so-called outgoing Sommerfeld radiation condition

$$
\lim _{R \rightarrow+\infty} R^{\frac{N}{2}}\left(u_{R}-i k n_{\infty} u\right)=0,
$$

uniformly; here, $N$ is the dimension of the space and $R$ is the radial variable. Under the same assumptions, Rellich condition is

$$
\lim _{R \rightarrow+\infty} \int_{\partial B_{R}}\left|u_{R}-i k n_{\infty} u\right|^{2} d \sigma=0,
$$

where $B_{R}$ is the ball of radius $R$ and $d \sigma$ is the surface element.

*Dipartimento di Matematica e Applicazioni, Università di Palermo, Via Archirafi 34, 90123 Palermo, Italy, (g.ciraolo@math.unipa.it). 
Both the conditions above say something about the geometry of the level sets of the phase of the solution: they are circles at leading order and their radii grow at a specific rate.

When such homogeneity condition at infinity of the refraction index is perturbed, it is unclear which should be the right geometry. Many papers have been written on this topic; we refer to Section 1 in [CM2] and references therein for a more detailed description of known results. Moreover, large perturbations of some fixed refraction index could change the rate of growing of the radii of the level sets of the phase function (that correspond to the right choice of the propagation constant in the radiation condition).

In this paper a step forward in those directions is given for the Helmholtz equation

$$
\Delta u+\left[k^{2} n(x)^{2}+p(x, z)\right] u=f, \quad(x, z) \in \mathbb{R}^{2},
$$

where $n$ is of the form

$$
n:= \begin{cases}n_{+}, & x>h, \\ n_{c o}(x), & |x| \leq h, \\ n_{-}, & x<-h ;\end{cases}
$$

here $k>0, n_{c o}(\cdot)$ is a real-valued function of bounded variation of the variable $x$, with $n_{+}, n_{-}, h$ positive constants and $p$ is a perturbing term satisfying certain hypothesis to be specified later.

Our work is motivated by the study of infinite open waveguides. Under the weakly guiding approximation (see $[\mathrm{SL}$ ), and for $p \equiv 0$, (4) describes the electromagnetic wave propagation in an optical or acoustical waveguide, where $k$ is the wavenumber and $n$ is the index of refraction. The peculiarity of the problem is the fact that the index of refraction $n$ is not a compact or small perturbation of the plane, and it may cause the appearance of guided modes, i.e. waves which propagates (each one with a different constant of propagation) in the $z$-direction without decaying. We will call radiating waves the waves that are not guided by the waveguide.

Our work is based on the knowledge of a Green's function $G$ for the nonperturbed $(p \equiv 0)$ Helmholtz equation

$$
\Delta u+k^{2} n(x)^{2} u=f, \quad(x, z) \in \mathbb{R},
$$

with $n$ given by (5); as done in [Wi], such a Green's function can be found by using Titchmarsh theory Ti] on eigenfunction expansions. We will make use of the results and notations in $\mathrm{MS}, \mathrm{CM} 1, \mathrm{CM} 2, \mathrm{Ci2}$ (where the case $n_{+}=n_{-}$ is deeply studied) and $\mathrm{Ci1}$ and $\mathrm{Ch}$ (for the expression of the Green's function in the general case).

Due to the presence of guided modes, the usual Sommerfeld radiation condition does not guarantee the uniqueness of solutions. The conditions proposed in [CM2, Xu1] and Xu2 provide the uniqueness for the Helmholtz equation in stratified media and they consist in a collection of Sommerfeld-like conditions for all guided components of the field and for the radiative component, each of them having its own wavenumber. In $\mathrm{Xu1}, \mathrm{Xu2}$, the author studies the case of a stratified medium with compactly supported inhomogeneities and gives a radiation condition in the spirit of (2). In CM2 and Ci2 analogous results are obtained by using an integral formulation of the radiation condition. In the present paper, we improve the mentioned results in the following sense: (i) we 
weaken the radiation condition (we use a radiation condition which is in the spirit of (31)); (ii) we consider inhomogeneities that can be extended to infinity in the direction of the waveguide but have to be small in some sense (see (H2) later).

We denote by $u_{0}$ the radiated part of the solution, $u_{1}, \ldots, u_{M}$ the guided ones and $\beta_{l}$ the propagation constant corresponding to $u_{l}, l=0,1, \ldots, M$, (see CM2 or Section 3 for a rigorous definition of $u_{l}$ and $\beta_{l}$ ). Then, the radiation condition introduced in CM2] (for the case $n_{c l}:=n_{+}=n_{-}$) is

$$
\int_{0}^{\infty} \int_{\partial \Omega_{R}}\left|\frac{\partial u_{0}}{\partial \nu}-i k n_{c l} u_{0}\right|^{2} d \ell d R+\sum_{l=1}^{M} \int_{0}^{\infty} \int_{\partial Q_{R}}\left|\frac{\partial u_{l}}{\partial \nu}-i \beta_{l} u_{l}\right|^{2} d \ell d R<+\infty,
$$

where $R=\sqrt{x^{2}+z^{2}}, \nu$ denotes the outward normal derivative and

$$
Q_{R}=\left\{(x, z) \in \mathbb{R}^{2}:|x|,|z| \leq R\right\}, \quad \Omega_{R}=\left\{(x, z) \in \mathbb{R}^{2}:[x]_{h}^{2}+z^{2} \leq R^{2}\right\},
$$

with

$$
[x]_{h}= \begin{cases}x+h, & x<-h \\ 0, & -h \leq x \leq h, \\ x-h, & x>h\end{cases}
$$

In $\mathrm{CM} 2$ it was also noticed that also the following radiation condition

$$
\sum_{l=0}^{M} \int_{0}^{\infty} \int_{\partial \Omega_{R}}\left|\frac{\partial u_{l}}{\partial \nu}-i \beta_{l} u_{l}\right|^{2} d \ell d R<+\infty
$$

still guarantees the existence and uniqueness of a solution for (4).

Both condition (7) and (10) say that the level sets of the phase of the radiating part of the solution are given by the sets $\partial \Omega_{R}$. An asymptotical approximation of the sets $\partial \Omega_{R}$ may be also used in the radiation conditions (in particular, it may be also a ball); we prefer to use the sets $\Omega_{R}$ because they lighten the analysis the asymptotic behaviour of the Green's function (see [CM2]). Regarding guided waves, (7) and (10) do not seem to distinguish which is the right geometry of the level sets, even if both of them ensure the uniqueness of the problem. We notice that guided waves are one dimensional solutions of the Helmholtz equation and thus the level sets of the phase function are just straight lines in the $x$-direction.

In this paper we provide a radiation condition of Sommerfeld-Rellich type which guarantees the uniqueness of solutions of (4), with $n$ given by (5) and where $p: \mathbb{R}^{2} \rightarrow \mathbb{C}$ is such that

(H1) $p(x, z)=0$ for $|x|>x_{0}$ for some positive $x_{0}$;

(H2) $p$ satisfies

$$
\sup _{(\xi, \zeta) \in \mathbb{R}^{2}} \int_{\mathbb{R}^{2}}|G(x, z ; \xi, \zeta) p(x, z)| d x d z<1 ;
$$

here, $G$ is the Green's function for the unperturbed stratified medium mentioned above (see Section 2 for more details). In particular, we are assuming that the perturbation is small in some sense and has compact support in the direction transversal to the waveguide. Our first result is the following: 
Theorem 1.1. Let $p$ satisfy assumptions (H1) and (H2). There exists at most one bounded solution of (4) satisfying

$$
\lim _{R \rightarrow+\infty} \int_{\partial \Omega_{R}}\left|\frac{\partial u_{0}}{\partial \nu}-i k n(x) u_{0}\right|^{2} d \ell+\sum_{l=1}^{M} \sqrt{R} \int_{\partial Q_{R}}\left|\frac{\partial u_{l}}{\partial \nu}-i \beta_{l} u_{l}\right|^{2} d \ell=0 ;
$$

here, $\nu$ denote the outward normal and $\Omega_{R}$ and $Q_{R}$ are given by (8).

We notice that Theorem 1.1 still holds if we consider the following radiation condition

$$
\lim _{R \rightarrow+\infty} \sum_{l=0}^{M} \int_{\partial \Omega_{R}}\left|\frac{\partial u_{l}}{\partial \nu}-i \beta_{l} u_{l}\right|^{2} d \ell=0 .
$$

We prefer to use (12) because it better describes the behaviour of guided modes: (i) the sets $Q_{R}$ suggest the geometry of the level sets of the guided modes (which are straight lines in the $x$-direction); (ii) the presence of $\sqrt{R}$ suggests that guided modes are lower dimensional solution of the Helmholtz equation.

The latter result of this paper concerns the existence of a solution satisfying (12). We shall assume that $p$ satisfies the following additional assumption:

(H3) $p \in L^{2}\left(\mathbb{R}^{2}\right)$ is such that

$$
\int_{\partial \Omega_{R}}|p|^{2} d \ell \leq c_{1} R^{-(3+2 \delta)},
$$

for some constant $c_{1}>0$ and $\delta>\frac{1}{2}$.

Then, our result is the following:

Theorem 1.2. Let $f$ and $p$ satisfy the assumptions (H1) and (H3) and assume that $p$ satisfies (H2), too. Then, there exist a unique bounded solution of (4) satisfying the radiation condition (12).

In particular, such a solution is the only bounded solution of the following integral equation:

$$
u(x, z)=\int_{\mathbb{R}^{2}} G(x, z ; \xi, \zeta)[f(\xi, \zeta)-p(\xi, \zeta) u(\xi, \zeta)] d \xi d \zeta .
$$

We notice that, if the waveguide is not rectilinear, the propagation constants $\beta_{l}$ become complex (see, for instance, $\overline{\mathrm{KNH}}$ ). Theorem 1.2 guarantees that, under the given assumptions, the propagation constants of the radiating and guided parts of the solution are (approximately) the same as in the unperturbed case and (12) still guarantee the existence and uniqueness of a solution. To the author's knowledge, it is not known if the exponent $\delta$ in (H3) can be improved (see also [Ei] for the case of non-stratified medium).

The paper is organized as follows.

In Section 2 we recall and prove some preliminary results which will be useful in the rest of the paper. 
Theorem 1.1 will be proved in Section 3. The technique used is in the spirit of classical results on the Helmholtz equation, in particular those contained in Mi1 and Mi2. Other techniques may be used to prove such theorem (for instance, the Limiting Absorption Principle, see [Hö] and $\mathrm{We}$ ); we shall include our proof of Theorem 1.1 because it is simple and direct.

In Section 4 we will prove Theorem [1.2. Here, a careful analysis of the asymptotic behaviour of the solution is done. Similar arguments for the free space case can be found in Ei.

We wish to mention that our approach can be generalized to stratified media in higher dimensions and in more general unbounded domains. Clearly, stratified media in higher dimensions may present more than one kind of stratification (in three dimensions, for instance, planar or cylindrical stratifications lead to different behaviours of the solution). Once a uniform asymptotic expansion of the Green's function is known, then it is possible to use the same technique in this paper and obtain analogous results. This will be the object of future work.

\section{Preliminaries}

In this section we recall and prove some results for the unperturbed Helmholtz equation, which will be useful in the rest of the paper. We notice that the case $n_{+}=n_{-}$has been deeply studied in [MS, [CM1, CM2] and [Ci2] and we refer to such works for a more extensive description of results and of the formulation of the outgoing Green's function.

By following [Wi] (see also Chapter 2 in [Ci1]), we write a solution $u$ of (6) in terms of a Green's function $G$, which is a superposition of solutions of the associated homogeneous equation:

$$
u(x, z)=\int_{\mathbb{R}^{2}} G(x, z ; \xi, \zeta) f(\xi, \zeta) d \xi d \zeta
$$

where

$$
G(x, z ; \xi, \zeta)=G_{0}(x, z ; \xi, \zeta)+\sum_{l=1}^{M} G_{l}(x, z ; \xi, \zeta)
$$

with

$$
G_{l}(x, z ; \xi, \zeta)=\frac{e^{i \beta_{l}|z-\zeta|}}{2 i \beta_{l}} e\left(x, \gamma_{l}\right) e\left(\xi, \gamma_{l}\right), \quad l=1, \ldots, M
$$

and

$$
\beta_{l}=\sqrt{k^{2} n_{*}^{2}-\gamma_{l}}, \quad l=1, \ldots, M .
$$

Here, $\gamma_{l}$ and $e\left(x, \gamma_{l}\right), l=1, \ldots, M$, are, respectively, the eigenvalues and eigenfunctions of the eigenvalue problem associated to (6) and obtained by separating the variables. In particular, by setting

$$
n_{*}=\max _{\mathbb{R}} n, \quad q(x)=k^{2}\left[n_{*}^{2}-n(x)^{2}\right],
$$

$e\left(x, \gamma_{l}\right)$ is the only $C^{1}$ solution of

$$
e^{\prime \prime}+\left[\gamma_{l}-q(x)\right] e=0, \quad \text { in } \mathbb{R},
$$


such that $\left\|e\left(\cdot, \gamma_{l}\right)\right\|_{L^{2}(\mathbb{R})}=1$ and which vanishes exponentially as $|x| \rightarrow+\infty$ (see [MS]). $G^{g}=\sum_{l=1}^{M} G_{l}$ represents the guided part of the Green's function, which involves the guided modes, i.e. the modes propagating mostly inside the waveguide; each $G_{l}, l=1, \ldots, M$, corresponds to a single guided mode.

In (17), $G_{0}$ is the part of the Green's function corresponding to the nonguided energy, i.e. the energy radiated outside the waveguide. In CM2 the case $n_{c l}:=n_{+}=n_{-}$has been carefully studied; in particular, it was proved that, for $\xi$ and $\zeta$ fixed, the following asymptotic expansions

$$
G_{0}=\mathcal{O}\left(R^{-\frac{1}{2}}\right), \quad \frac{\partial G_{0}}{\partial \nu}-i k n_{c l} G_{0}=\mathcal{O}\left(R^{-\frac{3}{2}}\right),
$$

uniformly as $R \rightarrow+\infty$ on the sets $\partial \Omega_{R}$, given by (8).

In the present paper, since we are allowing $n_{+}$and $n_{-}$to be different, we shall make use of the following result:

Lemma 2.1. Let $G_{0}$ be the Green's function mentioned above. Then, for $\xi$ and $\zeta$ fixed, we have

$$
\int_{\partial \Omega_{R}}\left|G_{0}\right|^{2} d \sigma=\mathcal{O}(1), \quad \int_{\partial \Omega_{R}}\left|\frac{\partial G_{0}}{\partial \nu}-i k n(x) G_{0}\right|^{2} d \sigma=\mathcal{O}\left(R^{-1}\right),
$$

where $\Omega_{R}$ is given by (8).

Proof. The results are a consequence of the (uniform) asymptotic expansion of $G_{0}$ for $R$ large. That can be done by following [Ch] and [CM2].

Lemma 2.2. Let $(x, z),(\xi, \zeta) \in \mathbb{R}^{2}$ and $\omega=(x-\xi, z-\zeta)$ with $|\omega| \leq 1$. There exists a positive constant $C_{1}$ independent on $x, z, \xi, \zeta$, such that

$$
\left|G_{0}(x, z ; \xi, \zeta)-\frac{1}{2 \pi} \log \right| \omega|| \leq C_{1} .
$$

Proof. In order to avoid heavy calculations, we carry out the scheme of the proof only for the case studied in [CM2], i.e. for $n_{c l}:=n_{+}=n_{-}$.

Instead of proving (22), we shall prove that $\left|G_{0}(x, z ; \xi, \zeta)-G_{F S}(x, z ; \xi, \zeta)\right|$ is uniformly bounded; here, we denoted by $G_{F S}$ the outgoing Green's function of the free-space case, i.e. $G_{F S}(x, z ; \xi, \zeta)=(4 \pi i)^{-1} H_{0}^{(1)}\left(k n_{c l}|\omega|\right)$, where $H_{0}^{(1)}$ is the zeroth-order Hankel function of the first kind.

The following integral representations will be useful for proving the lemma:

$$
\begin{aligned}
& G_{F S}(x, z ; \xi, \zeta)=\frac{1}{4 \pi i} \int_{\mathcal{C}} e^{i k n_{c l}[(x-\xi) \sin t+|z-\zeta| \cos t]} d t, \\
& G_{0}(x, z ; \xi, \zeta)=\int_{\mathcal{C}} g(x, \xi ; t) e^{i k n_{c l}\left([x]_{h} \sin t+|z-\zeta| \cos t\right)} d t,
\end{aligned}
$$

with $\mathcal{C}$ being the contour path shown in Fig 1 We shall not write the explicit expression of $g$ in (24) and we refer to formula (3.8) in CM2 for details since, here, we will make use only of the following asymptotic formula:

$$
g(x, \xi ; t)=\frac{1}{4 \pi i} e^{i k n_{c l}\left(\{x\}_{h}-\xi\right) \sin t}\left\{1+\frac{i}{2 k n_{c l} \sin t} \int_{\{\xi\}_{h}}^{\{x\}_{h}}\left[d^{2}-q(y)\right] d y\right\}+\mathcal{O}\left(\frac{1}{|\sin t|^{2}}\right),
$$






Figure 1: The contour $\mathcal{C}$.

which holds as $|t| \rightarrow \infty$ on $\mathcal{C}$, uniformly for $x \in \mathbb{R}$ and $\xi$ bounded (see Lemma A.2 in CM2 ); here, $\{x\}_{h}:=x-[x]_{h}$, with $[x]_{h}$ defined by (9). Lemma A.3 in CM2 assures that $g$ is bounded on $\mathcal{C}$. Thus, (22) follows straightforwardly from (23), (24), the above asymptotic expansion of $g$ and by observing that $\{x\}_{h}+[x]_{h}=x$.

\section{Proof of Theorem 1.1}

We consider a solution $u$ of (4) and define

$$
u_{l}(x, z)=e\left(x, \gamma_{l}\right) \int_{-\infty}^{+\infty} u(\xi, z) e\left(\xi, \gamma_{l}\right) d \xi, \quad l=1, \ldots, M
$$

and

$$
u_{0}(x, z)=u(x, z)-\sum_{l=1}^{M} u_{l}(x, z) .
$$

Lemma 3.1. Let $u$ be a weak solution of

$$
\Delta u+\left[k^{2} n(x)^{2}+p(x, z)\right] u=0, \quad(x, z) \in \mathbb{R}^{2},
$$

and define $u_{l}, l=0,1, \ldots, M$, as in (25). Then, $u_{l}$ is a weak solution of

$$
\Delta u_{l}+k^{2} n(x)^{2} u_{l}=-\psi_{l}, \quad l=0,1, \ldots, M,
$$

where we set

$$
\psi_{l}(x, z)=e\left(x, \gamma_{l}\right) \int_{\mathbb{R}} p(\xi, z) u(\xi, z) e\left(\xi, \gamma_{l}\right) d \xi, \quad l=1, \ldots, M,
$$

and

$$
\psi_{0}=p u-\sum_{l=1}^{M} \psi_{l} .
$$

Proof. The proof is analogous to part of the proof of Theorem 2.6 in [CM2] and hence is omitted. 
Lemma 3.2. Let $(\xi, \zeta) \in \mathbb{R}^{2}$ be fixed and $R$ be such that $(\xi, \zeta) \in \Omega_{R}$. Let $u$ be a solution of (26); then, we have the following identities:

$$
u_{0}(\xi, \zeta)+\int_{\Omega_{R}} G_{0} \psi_{0} d x d z=\int_{\partial \Omega_{R}}\left(u_{0} \frac{\partial G_{0}}{\partial \nu}-G_{0} \frac{\partial u_{0}}{\partial \nu}\right) d \ell,
$$

and

$$
e\left(\xi, \gamma_{l}\right) \int_{-R}^{R} e\left(s, \gamma_{l}\right) u(s, \zeta) d s+\int_{Q_{R}} G_{l} \psi_{l} d x d z=\int_{\partial Q_{R}}\left(u_{l} \frac{\partial G_{l}}{\partial \nu}-G_{l} \frac{\partial u_{l}}{\partial \nu}\right) d \ell
$$

for $l=1, \ldots, M$, and where $\psi_{l}, l=0, \ldots, M$, are given by (27).

Proof. Thanks to Lemma 3.1 we have that

$$
\int_{D}\left(u_{l} \Delta G_{l}-G_{l} \Delta u_{l}\right) d x d z=\int_{D}\left[u_{l}\left(\Delta G_{l}+k^{2} n(x)^{2} G_{l}\right)+G_{l} \psi_{l}\right] d x d z,
$$

for $l=0,1, \ldots, M$, and where $D$ is a (smooth enough) bounded domain (notice that, since $u$ is a weak solution of (26), by Theorem 8.8 in [GT], the integrals in (29) make sense). The above formula and the second Green's identity yield

$$
\int_{\partial D}\left(u_{l} \frac{\partial G_{l}}{\partial \nu}-G_{l} \frac{\partial u_{l}}{\partial \nu}\right) d \ell=\int_{D}\left[u_{l}\left(\Delta G_{l}+k^{2} n(x)^{2} G_{l}\right)+G_{l} \psi_{l}\right] d x d z,
$$

for $l=0,1, \ldots, M$.

Firstly, consider the case $l=0$. Thanks to Lemma 2.2. we know that $G_{0}$ has a singularity for $(x, z) \equiv(\xi, \zeta)$. We denote by $B_{\varepsilon}$ the ball centered in $(\xi, \zeta)$ of radius $\varepsilon$ and consider (29) with $D=\Omega_{R} \backslash B_{\varepsilon}$; thus, (28a) follows from (29), Lemma 2.2 and by taking the limit as $\varepsilon \rightarrow 0^{+}$.

Now, let $l=1, \ldots, M$ be fixed. From (18) it follows that $\Delta G_{l}+k^{2} n(x)^{2} G_{l}$ has a singularity for $z=\zeta$. By setting $D=Q_{R} \backslash\left\{(x, z) \in \mathbb{R}^{2}:|z-\zeta|<\varepsilon\right\}$ in (29), we obtain (28b) by taking the limit as $\varepsilon \rightarrow 0^{+}$.

Proof of Theorem 1.1. Let assume that $u^{1}$ and $u^{2}$ are two bounded solutions of (41) satisfying (12) and consider $u=u^{1}-u^{2}$. It is clear that $u$ is a bounded solution of (26) and satisfies (12). We write $u=u_{0}+u_{1}+\ldots+u_{M}$ as done in (25).

Let $(\xi, \zeta)$ be fixed and consider $R$ large enough such that $(\xi, \zeta) \in \Omega_{R}$. We set

$$
\Omega_{R}^{(l)}= \begin{cases}\Omega_{R}, & l=0, \\ Q_{R}, & l=1, \ldots, M,\end{cases}
$$

and

$$
J(R)=u_{0}(\xi, \zeta)+\sum_{l=1}^{M} e\left(\xi, \gamma_{l}\right) \int_{-R}^{R} e\left(s, \gamma_{l}\right) u(s, \zeta) d s+\sum_{l=0}^{M} \int_{\Omega_{R}^{(l)}} G_{l} \psi_{l} d x d z
$$


By summing up identities (28) for $l=0,1, \ldots, M$ and thanks to a simple manipulation, we obtain that

$$
J(R)=\sum_{l=0}^{M} \int_{\partial \Omega_{R}^{(l)}}\left[u_{l}\left(\frac{\partial G_{l}}{\partial \nu}-i \beta_{l} G_{l}\right)-G_{l}\left(\frac{\partial u_{l}}{\partial \nu}-i \beta_{l} u_{l}\right)\right] d \ell,
$$

where we set $\beta_{0}:=k n(x)$ (since it is not relevant in this proof, we are omitting the dependence of $\beta_{0}$ on $x$ ). Triangular and Cauchy-Schwartz inequalities yield

$$
\begin{aligned}
|J(R)| \leq \sum_{l=0}^{M}\left(\int_{\partial \Omega_{R}^{(l)}}\left|u_{l}\right|^{2} d \ell\right)^{\frac{1}{2}} & \left(\int_{\partial \Omega_{R}^{(l)}}\left|\frac{\partial G_{l}}{\partial \nu}-i \beta_{l} G_{l}\right|^{2} d \ell\right)^{\frac{1}{2}} \\
& +\left(\int_{\partial \Omega_{R}^{(l)}}\left|G_{l}\right|^{2} d \ell\right)^{\frac{1}{2}}\left(\int_{\partial \Omega_{R}^{(l)}}\left|\frac{\partial u_{l}}{\partial \nu}-i \beta_{l} u_{l}\right|^{2} d \ell\right)^{\frac{1}{2}} .
\end{aligned}
$$

Thanks to (25), Lemma 3.7 in [CM2] and Fubini-Tonelli's theorem, we obtain that

$$
\lim _{R \rightarrow+\infty} J(R)=u(\xi, \zeta)+\int_{\mathbb{R}^{2}} G(x, z ; \xi, \zeta) p(x, z) u(x, z) d x d z .
$$

From (18), (21) and since each $u_{l}, l=0,1, \ldots, M$, is bounded, we have that

$$
\int_{\partial \Omega_{R}^{(0)}}\left|G_{0}\right|^{2} d \ell=\mathcal{O}(1), \quad \int_{\partial \Omega_{R}^{(l)}}\left|G_{l}\right|^{2} d \ell=\mathcal{O}(R), \quad l=1, \ldots, M,
$$

and

$$
\int_{\partial \Omega_{R}^{(l)}}\left|u_{l}\right|^{2} d \ell=\mathcal{O}(R), \quad l=0,1, \ldots, M,
$$

as $R \rightarrow+\infty$; furthermore, from (18) we easily get that

$$
\int_{\partial \Omega_{R}^{(l)}}\left|\frac{\partial G_{l}}{\partial \nu}-i \beta_{l} G_{l}\right|^{2} d \ell, \quad l=1, \ldots, M,
$$

vanishes exponentially as $R \rightarrow+\infty$. From the above asymptotic estimates, (21) and since $u$ satisfies (12), it follows that the right hand side of (30) vanishes as $R \rightarrow+\infty$. Thus, by taking the limit for $R \rightarrow+\infty$ in (30), from (31) we have that

$$
u(\xi, \zeta)+\int_{\mathbb{R}^{2}} G(x, z ; \xi, \zeta) p(x, z) u(x, z) d x d z=0 .
$$

Since $u$ is bounded and by setting $L=\sup _{(x, z) \in \mathbb{R}^{2}}|u(x, z)|$, from the above formula we have

$$
L \leq L \sup _{(\xi, \zeta) \in \mathbb{R}^{2}} \int_{\mathbb{R}^{2}}|G(x, z ; \xi, \zeta) p(x, z)| d x d z,
$$

which, together with (11), implies that $L=0$, i.e. $u_{1}=u_{2}$. 


\section{Proof of Theorem 1.2}

The proof of Theorem 1.2 is a consequence of the following two lemmas.

Lemma 4.1. Let $\varphi \in L^{2}\left(\mathbb{R}^{2}\right)$ be a complex valued function satisfying (H1) and (H3). Then, the function

$$
w_{0}(x, z)=\int_{\mathbb{R}^{2}} G_{0}(x, z ; \xi, \zeta) \varphi(\xi, \zeta) d \xi d \zeta
$$

satisfies

$$
\lim _{R \rightarrow+\infty} \int_{\partial \Omega_{R}}\left|\frac{\partial w_{0}}{\partial \nu}-i k n(x) w_{0}\right|^{2} d \ell=0,
$$

with $\Omega_{R}$ given by (8).

Proof. We set $d(x, z)=\sqrt{[x]_{h}^{2}+z^{2}}$ and notice that $\partial \Omega_{R}, R>0$, are the level sets of $d$. Let $(x, z) \in \partial \Omega_{R}$ and set $\rho=R^{s}$, for some $0<s<1$. We have:

$$
\begin{aligned}
& \frac{\partial w_{0}}{\partial \nu}(x, z)-i k n(x) w_{0}(x, z)=\int_{\mathbb{R}^{2}}\left[\nabla G_{0} \cdot \nabla d(x, z)-i k n(x) G_{0}\right] \varphi(\xi, \zeta) d \xi d \zeta \\
& =\int_{\Omega_{\rho}}\left[\nabla G_{0} \cdot \nabla d(x, z-\zeta)-i k n(x) G_{0}\right] \varphi d \xi d \zeta \\
& \quad+\int_{\Omega_{\rho}} \nabla G_{0} \cdot \nabla[d(x, z)-d(x, z-\zeta)] \varphi d \xi d \zeta \\
& \quad+\int_{\Omega_{\rho}^{c} \backslash B_{1}(x, z)}\left[\nabla G_{0} \cdot \nabla d(x, z)-i k n(x) G_{0}\right] \varphi d \xi d \zeta \\
& \quad+\int_{B_{1}(x, z)} \nabla G_{0} \cdot \nabla d(x, z) \varphi d \xi d \zeta-\int_{B_{1}(x, z)} i k n(x) G_{0} \varphi d \xi d \zeta \\
& =I_{1}+I_{2}+I_{3}+I_{4}-I_{5} .
\end{aligned}
$$

Since the quantity $\nabla G_{0} \cdot \nabla d(x, z-\zeta)-i k n(x) G_{0}$ depends on $x, \xi$ and $z-\zeta$, from Lemma 2.1, we have that $\left|\nabla G_{0} \cdot \nabla d(x, z-\zeta)-i k n(x) G_{0}\right|=\mathcal{O}\left(R^{-\frac{3}{2}}\right)$ uniformly for $\xi$ and $\zeta$ bounded, and thus $\left|I_{1}\right|=\mathcal{O}\left(R^{-\frac{3}{2}}\right)$ (notice that (14) implies that $\left.\varphi \in L^{1}\left(\mathbb{R}^{2}\right)\right)$.

From (H1) we can assume that $\xi$ is bounded and, from Lemma 2.1, we infer that $\left|\nabla G_{0}\right|$ and $G_{0}$ are bounded in $\Omega_{\rho}$ for $R$ large enough. Thus, since

$$
|\nabla d(x, z)-\nabla d(x, z-\zeta)|=\mathcal{O}\left(\frac{\zeta}{R}\right),
$$

we have that $\left|I_{2}\right|$ is estimated (up to a multiplicative constant) by

$$
\frac{1}{R} \int_{\Omega_{R}}|\zeta||\varphi(\xi, \zeta)| d \xi d \zeta .
$$


Coarea formula (notice that $|\nabla d|=1$ ) and Hölder inequality yield

$$
\int_{\Omega_{\rho}}|\zeta||\varphi(\xi, \zeta)| d \xi d \zeta \leq \int_{0}^{\rho} r \int_{\partial \Omega_{r}}|\varphi| d \ell d r \leq \sqrt{2(\pi+h)} \int_{0}^{\rho} r^{\frac{3}{2}}\left(\int_{\partial \Omega_{r}}|\varphi|^{2} d \ell\right)^{\frac{1}{2}} d r
$$

and thus, from (14), we obtain that $I_{2}=\mathcal{O}\left(R^{s(1-\delta)-1}\right)$.

From Lemma 2.2 and since $|\nabla d|=1$, we obtain that (up to a multiplicative constant) $\left|I_{3}\right|$ is bounded by

$$
\int_{\Omega_{\rho}^{c}}|\varphi(\xi, \zeta)| d \xi d \zeta
$$

and thus $I_{3}=\mathcal{O}\left(R^{-s \delta}\right)$.

In order to estimate $I_{5}$, we use Hölder inequality and notice that $\left\|G_{0}\right\|_{L^{2}\left(B_{1}(x, z)\right)}$ is bounded by a constant independent on $\xi$ and $\zeta$, as follows from (22). Thus, from coarea formula and (14), we have

$$
\int_{B_{1}(x, z)}|\varphi(\xi, \zeta)|^{2} d \xi d \zeta \leq \int_{R-1}^{R+1} \int_{\partial \Omega_{r}}|\varphi|^{2} d \ell d r \leq 2 c_{1}(R-1)^{-(3+2 \delta)}
$$

which implies that $I_{5}=\mathcal{O}\left(R^{-\frac{3}{2}-\delta}\right)$.

By summing up the above estimates we find that

$$
\left|I_{1}+I_{2}+I_{3}+I_{5}\right|=\mathcal{O}\left(\max \left\{R^{-\frac{3}{2}}, R^{s(1-\delta)-1}, R^{-\delta s}, R^{-\frac{3}{2}-\delta}\right\}\right),
$$

as $R \rightarrow+\infty$, and thus

$$
\int_{\partial \Omega_{R}}\left|I_{1}+I_{2}+I_{3}+I_{5}\right|^{2} d \ell=\mathcal{O}\left(\max \left\{R^{-2}, R^{2 s(1-\delta)-1}, R^{-2 \delta s+1}, R^{-2(1+\delta)}\right\}\right),
$$

as $R \rightarrow+\infty$. By choosing $0<\varepsilon<1$ such that $\delta=\varepsilon+\frac{1}{2(1-\varepsilon)}$ and setting $s=1-\varepsilon$, we find that

$$
\lim _{R \rightarrow+\infty} \int_{\partial \Omega_{R}}\left|I_{1}+I_{2}+I_{3}+I_{5}\right|^{2} d \ell=0 .
$$

It remains to prove that

$$
\lim _{R \rightarrow+\infty} \int_{\partial \Omega_{R}}\left|I_{4}\right|^{2} d \ell=0
$$

We set $\omega=(x-\xi, z-\zeta)$. Working as in the proof of Lemma 2.2, we prove that $\left|\nabla G_{0} \cdot \omega\right|$ is bounded in $B_{1}(x, z)$ by a constant independent on $x, z, \xi, \zeta$. Thus, $\left|I_{4}\right|$ is estimated (up to a multiplicative constant) by

$$
\int_{B_{1}(x, z)} \frac{|\varphi(\xi, \zeta)|}{|\omega|} d \xi d \zeta
$$


where we set $p=(x-\xi, z-\zeta)$. From Hölder inequality, we estimate $\left|I_{4}\right|^{2}$ by

$$
\int_{B_{1}(x, z)} \frac{d \xi d \zeta}{|\omega|^{\frac{3}{2}}} \int_{B_{1}(x, z)} \frac{|\varphi(\xi, \zeta)|^{2}}{|\omega|^{\frac{1}{2}}} d \xi d \zeta=4 \pi \int_{B_{1}(x, z)} \frac{|\varphi(\xi, \zeta)|^{2}}{|\omega|^{\frac{1}{2}}} d \xi d \zeta .
$$

Fubini-Tonelli's Theorem yields

$$
\begin{aligned}
\int_{\partial \Omega_{R}} \int_{B_{1}(x, z)} \frac{|\varphi(\xi, \zeta)|^{2}}{|\omega|^{\frac{1}{2}}} d \xi d \zeta d x d z & \leq \int_{\partial \Omega_{R}}\left(\int_{\Omega_{R+1} \backslash \Omega_{R-1}} \frac{|\varphi(\xi, \zeta)|^{2}}{|\omega|^{\frac{1}{2}}} d \xi d \zeta\right) d x d z \\
& =\int_{\Omega_{R+1} \backslash \Omega_{R-1}}|\varphi(\xi, \zeta)|^{2}\left(\int_{\partial \Omega_{R}} \frac{1}{|\omega|^{\frac{1}{2}}} d x d z\right) d \xi d \zeta .
\end{aligned}
$$

Since

$$
\int_{\partial \Omega_{R}} \frac{1}{|\omega|^{\frac{1}{2}}} d x d z=\mathcal{O}(R)
$$

and from (14), we obtain that

$$
\int_{\partial \Omega_{R}}\left|I_{4}\right|^{2} d x d z=\mathcal{O}\left(R^{-2-2 \delta}\right)
$$

which completes the proof.

Lemma 4.2. Let $\varphi$ be as in Lemma 4.1. Then,

$$
w_{l}(x, z)=\int_{\mathbb{R}^{2}} G_{l}(x, z ; \xi, \zeta) \varphi(\xi, \zeta) d \xi d \zeta
$$

$l=1, \ldots, M$, satisfies

$$
\lim _{R \rightarrow+\infty} \sqrt{R} \int_{Q_{R}}\left|\frac{\partial w_{l}}{\partial \nu}-i \beta_{l} w_{l}\right|^{2} d \ell=0 .
$$

Proof. Let $(x, z) \in \partial Q_{R}$, with $|x|=R$. Thus, $\frac{\partial}{\partial \nu}=\frac{\partial}{\partial|x|}$ and it is easy to show that

$$
\begin{aligned}
\left|\frac{\partial w_{l}}{\partial \nu}-i \beta_{l} w_{l}\right| & =K_{l}\left|e\left(x, \gamma_{l}\right)\right| \int_{\mathbb{R}^{2}}\left|e\left(\xi, \gamma_{l}\right) \varphi(\xi, \zeta)\right| d \xi d \zeta \\
& \leq K_{l}\left|e\left(x, \gamma_{l}\right)\right|\left\|e\left(\cdot, \gamma_{l}\right)\right\|_{L^{\infty}(\mathbb{R})}\|\varphi\|_{L^{1}\left(\mathbb{R}^{2}\right)}
\end{aligned}
$$

with $K_{l}, l=1, \ldots, N$, positive constants; since $\left|e\left(x, \gamma_{l}\right)\right|$ vanishes exponentially as $|x| \rightarrow+\infty$, we obtain that

$$
\lim _{R \rightarrow+\infty} \sqrt{R} \int_{Q_{R} \cap\{|x|=R\}}\left|\frac{\partial w_{l}}{\partial \nu}-i \beta_{l} w_{l}\right|^{2} d \ell=0 .
$$

Now, we consider $(x, z) \in \partial Q_{R}$, with $|z|=R$ (thus $\left.\frac{\partial}{\partial \nu}=\frac{\partial}{\partial|z|}\right)$. We write

$$
\frac{\partial w_{l}}{\partial \nu}-i \beta_{l} w_{l}=\int_{\{|\zeta|<R\}}+\int_{\{|\zeta| \geq R\}}\left[\frac{\partial G_{l}(x, z ; \xi, \zeta)}{\partial|z|}-i \beta_{l} G_{l}(x, z ; \xi, \zeta)\right] \varphi(\xi, \zeta) d \xi d \zeta .
$$


From (18) it follows that the first integral on the right hand side vanishes, since there $|z|>|\zeta|$. We estimate the second integral as follows:

$$
\int_{\{|\zeta| \geq R\}}\left|\frac{\partial G_{l}}{\partial|z|}-i \beta_{l} G_{l}\right||\varphi| d \xi d \zeta \leq\left\|e\left(\cdot, \gamma_{l}\right)\right\|_{L^{\infty}(\mathbb{R})}\left|e\left(x, \gamma_{l}\right)\right| \int_{\mathbb{R}^{2} \backslash \Omega_{R}}|\varphi(\xi, \zeta)| d \xi d \zeta .
$$

Since $\varphi$ satisfies (H1), coarea formula and Hölder inequality yield

$$
\int_{\mathbb{R}^{2} \backslash \Omega_{R}}|\varphi(\xi, \zeta)| d \xi d \zeta=\int_{R}^{+\infty} \int_{\partial \Omega_{r}}|\varphi| d \ell d r \leq \sqrt{2 \pi} \int_{R}^{+\infty} \sqrt{r}\left(\int_{\partial \Omega_{r}}|\varphi|^{2} d \ell\right)^{\frac{1}{2}} d r ;
$$

from (14) we obtain that

$$
\int_{R}^{+\infty} \sqrt{r}\left(\int_{\partial \Omega_{r}}|\varphi|^{2} d \ell\right)^{\frac{1}{2}} d r \leq \frac{\sqrt{c_{1}}}{\delta} R^{-\delta}
$$

and then

$$
\left|\frac{\partial w_{l}}{\partial \nu}-i \beta_{l} w_{l}\right| \leq \frac{\sqrt{2 \pi c_{1}}}{\delta}\left\|e\left(\cdot, \gamma_{l}\right)\right\|_{L^{\infty}(\mathbb{R})}\left|e\left(x, \gamma_{l}\right)\right| R^{-\delta} .
$$

Since $\left\|e\left(\cdot, \gamma_{l}\right)\right\|_{2}=1$, we have that

$$
\begin{aligned}
\int_{Q_{R} \cap\{|z|=R\}}\left|\frac{\partial w_{l}}{\partial \nu}-i \beta_{l} w_{l}\right|^{2} d \ell & \leq \frac{2 \pi c_{1}}{\delta^{2}}\left\|e\left(\cdot, \gamma_{l}\right)\right\|_{L^{\infty}(\mathbb{R})} R^{-2 \delta} \int_{-R}^{R}\left|e\left(x, \gamma_{l}\right)\right|^{2} d x \\
& \leq \frac{2 \pi c_{1}}{\delta^{2}}\left\|e\left(\cdot, \gamma_{l}\right)\right\|_{L^{\infty}(\mathbb{R})} R^{-2 \delta}
\end{aligned}
$$

from the above estimate and since $\delta>\frac{1}{2}$, we obtain that

$$
\lim _{R \rightarrow+\infty} \sqrt{R} \int_{Q_{R} \cap\{|z|=R\}}\left|\frac{\partial w_{l}}{\partial \nu}-i \beta_{l} w_{l}\right|^{2} d \ell=0,
$$

which completes the proof.

Proof of Theorem 1.2. Firstly we prove that $u$ is bounded and then show that it satisfies (12).

We notice that, if we prove that $\int_{\mathbb{R}^{2}} G f$ is bounded, then we conclude that $u$ is bounded, as follows from (11) and a contraction mapping theorem. In order to prove that, we write

$$
\int_{\mathbb{R}^{2}} G(x, z ; \xi, \zeta) f(\xi, \zeta) d \xi d \zeta=\int_{B_{1}(x, z)}+\int_{\mathbb{R}^{2} \backslash B_{1}(x, z)} G(x, z ; \xi, \zeta) f(\xi, \zeta) d \xi d \zeta .
$$

Hölder inequality and (22) imply that the first integral on the right hand side is bounded by the $L^{2}$ norm of $f$ multiplied by a constant independent on $(x, z)$. We notice that the assumptions on $f$ imply that $f \in \mathrm{L}^{1}\left(\mathbb{R}^{2}\right)$; since $G$ is bounded 
outside $B_{1}(x, z)$ (as follows from Lemmas 2.1 and 2.2), we obtain the boundness of the second integral on the right hand side in (32) and conclude that $u$ is bounded.

It remains to prove that $u$ satisfies the radiation condition (12). We write (15) as

$$
u(x, z)=\sum_{l=0}^{M} \int_{\mathbb{R}^{2}} G_{l}(x, z ; \xi, \zeta)[f(\xi, \zeta)-p(\xi, \zeta) u(\xi, \zeta)] d \xi d \zeta ;
$$

since $u$ is bounded, the conclusion follows straightforwardly from the assumptions on $f$ and $p$ by using Lemmas 4.1 and 4.2

Remark 4.3. In Theorem 1.2, we assumed that $f$ and $p$ satisfy (H1). Such an assumption is due to the fact that, for proving Lemma 4.1, we need an uniform asymptotic expansion of the far-field of $G_{0}$, that we indeed have only if we assume that $\xi$ is bounded. We notice that, in Lemma 4.2 the assumption can be omitted, as it is clear from its proof.

\section{References}

[Ch] T. Christiansen, Scattering theory for perturbed stratified media, Journal d'Analyse Mathématique, 76 (1998), pp. 1-44.

[Ci1] G. CiraOlo, Non-rectilinear waveguides: analytical and numerical results based on the Green's function, Ph.D. Thesis, http://www.math.unipa.it/〜g.ciraolo/ .

[Ci2] G. Ciraolo, A method of variation of boundaries for waveguide grating couplers, Applicable Analysis, 87 (2008), no.9, pp. 1019-1040.

[CM1] G. Ciraolo and R. Magnanini, Analytical results for 2-D nonrectilinear waveguides based on the Green's function, Math. Methods Appl. Sci., 31 (2008), no.13, pp. 1587-1606.

[CM2] G. Ciraolo - R. Magnanini, A radiation condition for uniqueness in a wave propagation problem for 2-D open waveguides, Math. Methods Appl. Sci., ?? (2009), no.??, pp. ??.

[Ei] D. EIDUs, The principle of limiting absorption, Thirteen papers on functional analysis and partial differential equations, American Mathematical Society Translations, Series 2, 47 (1965), pp. 157 - 191.

[GT] D. Gilbarg And N. S. Trudinger, Elliptic partial differential equations of second order. Springer-Verlag, 1983.

[Hö] L. HöRMANDER, The analysis of linear partial differential operators. II. Differential operators with constant coefficients. Classics in Mathematics. Springer-Verlag, Berlin, 2005.

$[\mathrm{KNH}]$ E. M. Kartchevski, A. I. Nosich and G. W. Hanson, Mathematical analysis of the generalized natural modes of an inhomogeneous optical fiber, SIAM J. Appl. Math., 65 (2005), no. 6, pp. 2033 - 2048. 
[Mi1] W. L. MIRANKER, Uniqueness and representation theorems for solutions of $\Delta u+k^{2} u=0$ in infinite domains, Journal of Mathematics and Mechanics, 6 (1957), pp. $847-854$.

[Mi2] W. L. Miranker, The reduced wave equation in a medium with a variable index of refraction. Comm. Pure Appl. Math., 10 (1957) pp. 491-502.

[MS] R. Magnanini And F. Santosa, Wave propagation in a 2-D optical waveguide, SIAM J. Appl. Math., 61 (2001) 1237 - 1252.

[Rel] F. RELLICH, Über das asymptotische Verhalten del Lösungen von $\Delta u+\lambda u=0$ in unendlichen Gebieten, Jahresbericht der Deutschen Mathematiker-Vereinigung, 53 (1943), pp. 57 - 65 .

[So1] A. Sommerfeld, Die Greensche Funktion der Schwingungsgleichung, Deutsche Math.-Ver. 21 (1912), pp. 309-353.

[So2] A. Sommerfeld, Partial differential equations in physics, Academic Press, 1949.

[SL] A. W. SNyder And D. Love, Optical Waveguide Theory, Chapman and Hall, London, 1974.

[Ti] E. C. Titchmarsh, Eigenfunction expansions associated with secondorder differential equations. Oxford at the Clarendon Press, Oxford, 1946.

[We] R. WEDER, Spectral and scattering theory for wave propagation in perturbed stratified media. Applied Mathematical Sciences, 87. SpringerVerlag, New York, 1991.

[Wi] C. H. Wilcox, Sound propagation in stratified fluids, Applied Mathematical Sciences, 50. Springer-Verlag, New York, 1984.

[Xu1] Y. XU, Scattering of acoustic waves by an obstacle in a stratified medium. Partial differential equations with real analysis, Pitman Res. Notes Math. Ser., 263 (1992), pp. 147-168.

[Xu2] Y. XU, Radiation condition and scattering problem for time-harmonic acoustic waves in a stratified medium with a nonstratified inhomogeneity, IMA J. Appl. Math. 54 (1995), no. 1, pp. 9-29. 